\title{
Collaboration Versus Cheating
}

\author{
Reducing Code Plagiarism in an Online MS Computer Science Program
}

\author{
Tony Mason* \\ Georgia Institute of Technology \\ fsgeek@gatech.edu
}

\author{
Ada Gavrilovska \\ Georgia Institute of Technology \\ ada@cc.gatech.edu
}

\author{
David A. Joyner \\ Georgia Institute of Technology \\ david.joyner@gatech.edu
}

\begin{abstract}
We outline how we detected programming plagiarism in an introductory online course for a master's of science in computer science program, how we achieved a statistically significant reduction in programming plagiarism by combining a clear explanation of university and class policy on academic honesty reinforced with a short but formal assessment, and how we evaluated plagiarism rates before and after implementing our policy and assessment.
\end{abstract}

\section{CCS CONCEPTS}

- Social and professional topics $\rightarrow$ Computer science education; CS1; Student assessment;

\section{KEYWORDS}

MOSS, Academic Honesty, Honor Codes, Plagiarism, MOOC, Online, OMSCS

\section{ACM Reference Format:}

Tony Mason, Ada Gavrilovska, and David A. Joyner. 2019. Collaboration Versus Cheating: Reducing Code Plagiarism in an Online MS Computer Science Program. In Proceedings of the 50th ACM Technical Symposium on Computer Science Education (SIGCSE '19), February 27-March 2, 2019, Minneapolis, MN, USA. ACM, New York, NY, USA, 7 pages. https://doi.org/ $10.1145 / 3287324.3287443$

\section{INTRODUCTION}

Academic honesty is critical for those engaged in research. Because we build upon the work of others, we must be able to rely on the integrity of work done by others:

\begin{abstract}
As a society, we rely on the academic and journalistic integrity of other people's work. The whole point of academic research is to share knowledge with others and learn from one another. Since knowledge and ideas are the primary product produced by academic communities, it is essential that this knowledge is accurate and gives credit to those who created it [42].
\end{abstract}

\footnotetext{
*Also affiliated with the University of British Columbia (fsgeek@cs.ubc.ca)
}

Permission to make digital or hard copies of all or part of this work for personal or classroom use is granted without fee provided that copies are not made or distributed for profit or commercial advantage and that copies bear this notice and the full citation on the first page. Copyrights for components of this work owned by others than the author(s) must be honored. Abstracting with credit is permitted. To copy otherwise, or republish, to post on servers or to redistribute to lists, requires prior specific permission and/or a fee. Request permissions from permissions@acm.org.

SIGCSE '19, February 27-March 2, 2019, Minneapolis, MN, USA

(C) 2019 Copyright held by the owner/author(s). Publication rights licensed to ACM.

ACM ISBN 978-1-4503-5890-3/19/02 ..\$15.00

https://doi.org/10.1145/3287324.3287443
The concept of intellectual integrity is not restricted to academic work; dishonest behavior spills over into the non-academic world. Cheating is a difficult habit to break, and forms of cheating (such as plagiarism) spread easily when left unchallenged [12].

While instructors for complex technical classes focus on promoting mastery, students often focus on achieving the highest possible marks. When they have not achieved mastery of the material, they may submit the work of others as their own. The issue of academic honesty in programming classes is not a new problem, having been reported early in the history of computer science education[24]. The problem of plagiarism is complicated by the fact that the division between collaboration and cheating for programming is not clear; modern work practice is often to modify existing code to fit the current need.

Plagiarism in which students copied the work of those who took the same course in earlier semesters was problematic in our online program, which grants a master of science degree in computer science to students who complete it. The program began in 2014, and all courses and student interactions are conducted online [23]. As of Spring 2018 there were 6,365 students enrolled, with most (70.2\%) being US citizens or permanent residents; $85.1 \%$ were men, and $14.8 \%$ were from under-represented minorities. Most students were enrolled in a single class, in which total course enrollments reached $8,737 .^{1}$

One challenge in delivering computer science classes at this scale is implementing an effective formative analysis of students' comprehension without unduly burdening the instructional team. Some classes in our program create unique projects each term, but this is not practical for our class, as we provide automated feedback via a "black box" testing mechanism, which requires considerable effort to modify, let alone rewrite from scratch each semester. Similarly, we found that a strict enforcement mechanism was effective in suppressing the repeated instances of academic dishonesty from the same students, but substantial effort was required to follow the due process requirements of the university; we observed that some instructors ignored problems rather than dedicate the considerable effort required to vigorously pursue them.

We were motivated to conduct this research because we observed that plagiarism in our course was an increasing problem. We discussed this situation internally, reviewed techniques for mitigating the issue, and even implemented several suggested mechanisms. While we found that rapid response and enforcement was effective for subsequent projects, we sought a mechanism that would discourage plagiarism before it became a burden on the instructional team. In addition, fewer cases up front would make it easier to enforce the class policy simply because there would be fewer such cases to report to the university.

\footnotetext{
${ }^{1}$ https://www.omscs.gatech.edu/prospective-students/numbers
} 


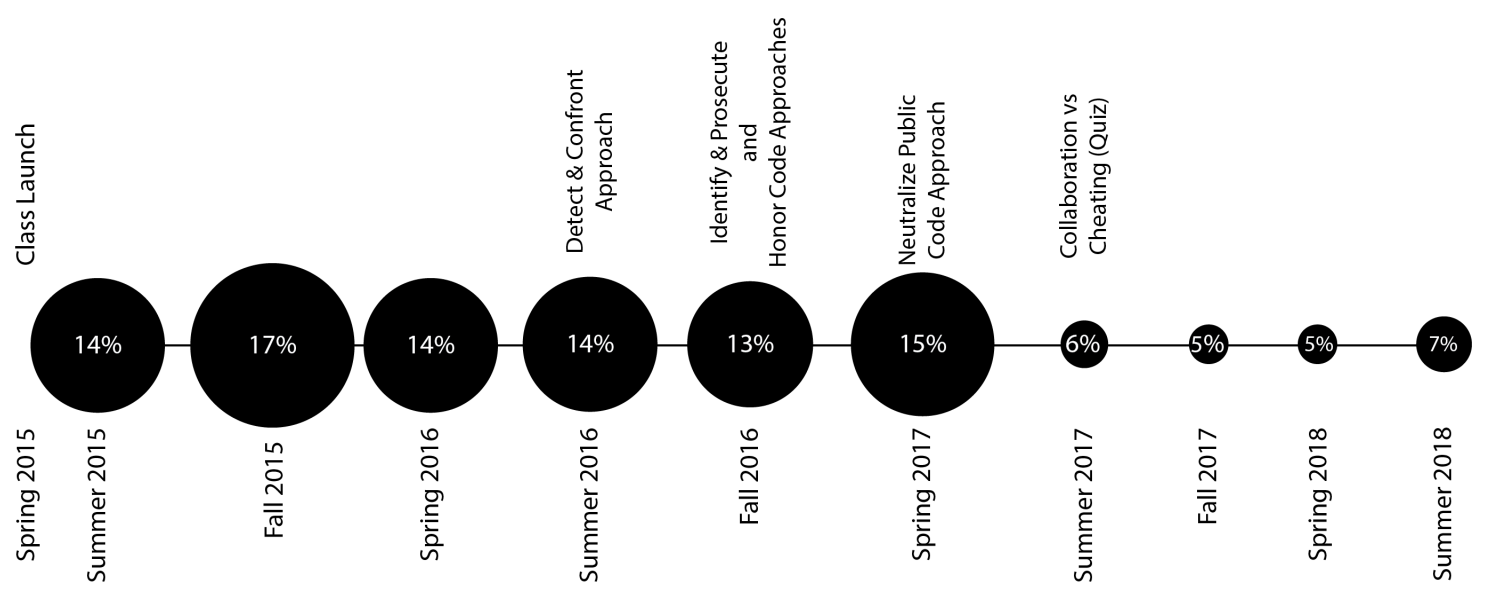

Figure 1: Course timeline

Course timeline and percentage of unique students detected via automatic plagiarism checks. See Table 5 for detailed data. A student flagged multiple times is only counted once.

In Summer 2017 we introduced a single change: a formal assessment, given via our learning management system (LMS), that spelled out the expected behavior from students in our class, why we viewed it as an important aspect of the course, and the potential ramifications for students that violated the policy. We observed a substantial drop in the number of detected cases. At that point we decided to perform a more rigorous evaluation of our results. This led to a thorough review of our historical data, as well as an ongoing review of subsequent classes, which allowed us to determine whether we were, in fact, observing a valid trend. We now have four semesters of data since we first implemented the formal assessment. We have observed a statistically significant decrease in the rates of plagiarism in the courses based upon our approach of using a quiz. Figure 1 shows our measured rate of plagiarism over time and the effect of our change beginning in Summer 2017. Table 4 demonstrates our claim this represents a statistically significant decrease in plagiarism.

Our approach combines a clear definition of the course expectations and the university's honor code. This is explained in detail to the students in the form of a formal assessment exercise. ${ }^{2}$ This approach, a low-effort mechanism for clearly communicating expectations in an instructional setting, decreased our objectively measured plagiarism rate. It also represents a novel "middle ground" between prior work that showed honor codes were not effective in online classes and one that showed that a comprehensive course in academic honesty, with evaluation, was effective. We suggest this approach can be applied successfully to other, similar courses.

\section{BACKGROUND}

Our course includes two substantial programming projects, which are generally reused each semester with only minor revisions. While some classes in the program do change their projects from semester to semester, it is not feasible for our class to change our course projects.

\footnotetext{
${ }^{2}$ https://github.com/fsgeek/collaboration-versus-cheating
}

Students are given initial "starter" code with instructions on the project requirements. They are encouraged to collaborate with one another, but to complete and submit their own work. Programs are submitted to a test framework that provides feedback but not final grades. Students are required to submit a README file with the project, explaining their understanding of the project, the challenges they experienced, and the external sources they used.

\subsection{Plagiarism}

For the purposes of our evaluation, we determined the presence of plagiarism using the results of MOSS[2], which is a standard tool used in plagiarism detection for programming courses in our programs. We invoked MOSS using -l c -n $1000-d-c$ " "<project name >' ' -b <template> ... -b <template>. This set of options tells MOSS that the language (-1) is $C[32]$, requests the top 1000 "hits" $(-n)$, designates files in the same directory $(-d)$ as part of the same program, adds a unique name to the top of the MOSS output $(-c)$, and ensures that the starter code $(-b)$ is not itself assessed for plagiarism.

In a normal class execution, we manually assess each case of suspected plagiarism. MOSS is a tool to identify potential cases; results are suggestive of plagiarism, but not definitive. To make a referral to the university, we must be sure that the reported offense is defensible.

In evaluating the effectiveness of our instructional approach for plagiarism reduction, we rejected using the manual evaluation method in order to avoid injecting additional bias into our analysis. Instead, we chose a definitive cut-off of $30 \%$ MOSS similarity. We chose this value because, in reviewing the cases considered by the instructional team, we noted that all instances at or above $30 \%$ had been referred by the instructional staff, no cases below $20 \%$ were referred, and some cases between $20 \%$ and $30 \%$ were referred. We were confident that a demonstrable decrease in the plagiarism rate at this level was strongly supportive of an effective reduction scheme, even if there were a small number of false positive results. 
Table 1: Project 1 Submission \& Plagiarism Data

Project 1 submissions consist of four distinct units; each unit is independently analyzed. Per semester, we report the total number of submissions and the percentage flagged for plagiarism (as defined in §2.1).

\begin{tabular}{|c|c|c|c|c|c|c|c|c|}
\hline \multirow{2}{*}{ Semester } & \multicolumn{2}{|c|}{ PR1-A } & \multicolumn{2}{|c|}{ PR1-B } & \multicolumn{2}{|c|}{ PR1-C } & \multicolumn{2}{|c|}{ PR1-D } \\
\hline & Submissions & $\%$ Plagiarism & Submissions & $\%$ Plagiarism & Submissions & $\%$ Plagiarism & Submissions & $\%$ Plagiarism \\
\hline Summer 2015 & 152 & 3.95 & 145 & 4.14 & 121 & 3.31 & 129 & 5.43 \\
\hline Fall 2015 & 200 & 9.50 & 194 & 7.73 & 190 & 4.74 & 191 & 6.81 \\
\hline Spring 2016 & 174 & 5.75 & 170 & 7.06 & 167 & 3.59 & 162 & 4.94 \\
\hline Summer 2016 & 150 & 1.33 & 153 & 4.58 & 124 & 4.84 & 123 & 4.07 \\
\hline Fall 2016 & 183 & 6.01 & 183 & 6.56 & 156 & 3.85 & 155 & 5.77 \\
\hline Spring 2017 & 140 & 7.86 & 140 & 10.71 & 119 & 10.08 & 121 & 12.40 \\
\hline Summer 2017 & 77 & 1.30 & 70 & 0.00 & 67 & 2.99 & 68 & 0.00 \\
\hline Fall 2017 & 224 & 2.68 & 225 & 1.78 & 203 & 2.46 & 209 & 1.44 \\
\hline Spring 2018 & 274 & 4.01 & 281 & 4.27 & 235 & 1.70 & 245 & 2.45 \\
\hline Summer 2018 & 172 & 3.49 & 171 & 5.26 & 160 & 1.88 & 166 & 1.81 \\
\hline
\end{tabular}

Although our initial choice of $30 \%$ was based on our ad hoc analysis of the cases that we had reviewed, prior work suggests this was a reasonable approach, based upon the low likelihood of a false positive over a large number of MOSS tokens[55]. The projects in our class typically include several hundred lines of $\mathrm{C}$ code written by students. As a result, the total number of unique tokens evaluated by MOSS is quite substantial, and false positives are less likely.

The original class practice involved evaluating plagiarism using only intra-semester data, but we observed that incorporating inter-semester data revealed $20 \%$ more cases of plagiarism. With this in mind, we converted to using a full cross-semester analysis: we compared all student submissions between Summer 2015 and Summer 2018 (10 semesters). In cases where trying to evaluate all submissions over time did not work - MOSS simply never returned results - we compared the current semester's submissions against individual prior years' submissions, combining the results at the end for the current semester. We found this result consistent with prior work [44].

\subsection{Course Messaging (Pre-Summer 2017)}

The inaugural semester of the course was Spring 2015; there was no prior body of work from which students could draw. Beyond making students aware of the existing university honor code, we did not raise the issue of plagiarism to the class during that semester.

In Spring 2016 we noticed indications of plagiarism and began to investigate. Our first course of action was to augment the syllabus to more clearly explain the course standards for plagiarism. We also offered amnesty to students who came forward to admit their plagiarism, but found amnesty completely ineffective; none of the students from the suspected cases came forward. Instead, students who had used small online examples of code came forward.

In Spring 2017 we took aggressive action by quickly completing a review of the code submissions shortly after students completed each project. When we identified specific cases of suspected plagiarism, we scheduled meetings to discuss our findings with the students. We made contact via the online student forum system (Piazza) and conducted online meetings (via WebEx or BlueJeans).
Many students ignored our requests to discuss their assignments. Of those who spoke with us, some admitted to plagiarism and some denied it. All cases were submitted to the university under its process for handling the issue. The plagiarism rate between the first and second project plummeted. However, we estimate that each case we prosecuted required more than five hours of instructional team time. This approach, while effective, was too labor intensive to scale for a large class.

\subsection{Collaboration versus Cheating}

Beginning in Summer 2017, we augmented the existing information about course expectations, the academic honesty policy, and penalties for violating this policy with an additional reinforcement mechanism: a quiz, administered using the university's LMS, that required the students' active acknowledgement that they understood and agreed to the policy. The initial results for Summer 2017 were encouraging: the number of cases of plagiarism noticeably decreased. However, the class size for that semester was the smallest to date. While we were optimistic, we decided it was premature to declare victory.

Subsequent semesters supported our finding that there was a statistically significant decrease in the rate of plagiarism in Summer 2017. We discuss our findings in $\S 3$ and our analysis in $\S 4$.

\section{EVALUATION}

Each project in our course consists of discrete parts: Project 1 has four and Project 2 has two. Each component is individually submitted for grading, and we evaluate each distinct component using MOSS. Table 1 reports our results for Project 1 from Summer 2015 through Summer 2018. Table 2 reports our results for Project 2 from Summer 2015 through Fall 2017, excluding Spring 2017, as that semester we implemented a different, aggressive-prosecution model. Table 3 summarizes our results for all presentations of the course. Table 5 summarizes our results for all unique students identified as plagiarizing assignments in a given semester. 
Table 2: Project 2 Submission \& Plagiarism Data

Project 2 consists of two separate submission units. Per semester, we report the total number of submissions and the percentage flagged for plagiarism (as defined in §2.1).

\begin{tabular}{rcccc}
\hline \multicolumn{1}{c}{ Semester } & \multicolumn{2}{c}{ Part 1 } & \multicolumn{2}{c}{ Part 2 } \\
& Subm issions & \%Plag iarism & Subm issions & \%Plag iarism \\
\hline Summer 2015 & 136 & 7.35 & 132 & 7.58 \\
Fall 2015 & 147 & 8.16 & 132 & 8.33 \\
Spring 2016 & 142 & 1.41 & 140 & 2.86 \\
Summer 2016 & 135 & 8.11 & 126 & 7.94 \\
Fall 2016 & 155 & 6.45 & 146 & 8.90 \\
Spring 2017 & 122 & 4.10 & 122 & 3.28 \\
Summer 2017 & 71 & 0.00 & 68 & 2.94 \\
Fall 2017 & 203 & 0.99 & 196 & 0.00 \\
Spring 2018 & 251 & 0.00 & 238 & 0.84 \\
Summer 2018 & 164 & 0.00 & 159 & 3.14 \\
\hline
\end{tabular}

\subsection{Effectiveness}

To evaluate our "Collaboration versus Cheating" approach, we analyze each separate part of a submitted project by performing a one-tailed two-sample t-test, assuming unequal variances. This approach is appropriate for this evaluation because we are interested only in interventions that yield lower plagiarism rates. We are testing distinct groups of students, but with similar backgrounds (see $\S 4.2$ for further discussion). We tested against the null hypothesis with a $95 \%$ confidence $(p<0.05)$.

In evaluating Project 1 cases, we use data from Summer 2015 through Summer 2018. Data from Summer 2015 through Spring 2017 represent the data prior to the introduction of this approach, while those from Summer 2017 through Summer 2018 represent the data since the implementation of this approach. We omit Spring 2017 Project 3 data, as it relates to the strong success of a rapid response scheme, where the instructional team reached out to Project 1 students. No other semester used this approach; it does not surprise us that aggressive enforcement is generally successful.

Our interpretation of these results is that the samples in question are unlikely to come from the same population $(p<0.05)$. We conclude that our intervention in this case was significant. However, we acknowledge the possibility that some other differences account for these changes (this is further discussed in $§ 4$ ).

Independently, we examine the number of unique students identified per semester. These data are shown in Table 5. The change is clearly visible from the table. We perform a comparable t-test on these results, comparing the Summer 2015 through Spring 2016 results against the Summer 2016 through Summer 2018 results, and once again note that these results are unlikely to come from the same population $(p<0.05)$.

\subsection{Resource Requirements}

An important consideration for us regarding the role of course instructors is the level of resources required for implementing antiplagiarism techniques. The time required to add the quiz to the LMS is nominal, and the material can be copied from one semester to another. The post we add to our student engagement forum (Piazza) is also copied from the prior semester. The total effort required for this is less than one hour and is independent of the number
Table 3: Aggregate Plagiarism Results

MOSS-reported similarity across all semesters: breakdown by project/sub-project, reporting number of flagged submissions, percentage (of total), plus average similarity $\%$ and average line count similarity (with standard deviation).

\begin{tabular}{lccccccc}
\hline & & \multicolumn{3}{c}{ Plagiarism } & \multicolumn{4}{c}{ MOSS } \\
\cline { 3 - 7 } Project & Total Submissions & Detected & Percent & $\begin{array}{c}\text { Average } \\
\%\end{array}$ & $\begin{array}{c}\text { Standard } \\
\text { Deviation }\end{array}$ & $\begin{array}{c}\text { Average } \\
\text { Lines }\end{array}$ & $\begin{array}{c}\text { Standard } \\
\text { Deviation }\end{array}$ \\
\hline PR1-A & 1647 & 83 & 5.04 & 13.01 & 15.47 & 44.33 & 50.30 \\
PR1-B & 1623 & 92 & 5.67 & 13.11 & 16.96 & 31.19 & 35.23 \\
PR1-C & 1409 & 57 & 4.05 & 15.77 & 11.51 & 38.04 & 36.75 \\
PR1-D & 1475 & 69 & 4.68 & 18.10 & 15.01 & 41.81 & 59.94 \\
PR2-A & 1509 & 52 & 3.45 & 10.76 & 11.16 & 30.82 & 25.00 \\
PR2-B & 1453 & 61 & 4.20 & 8.21 & 15.41 & 62.77 & 100.14 \\
\hline
\end{tabular}

of students in the class. Responses to follow-up questions from students and clarification of the policy require approximately an additional hour of time. Fewer than a dozen students asked followup questions on average; most questions consisted only of one or two paragraphs and were asked via Piazza[43] so that the answers were visible to the entire class.

The time required for the students to complete the evaluation was generally less than 15 minutes.

In addition, our work for evaluating the plagiarism activity has led us to automate much of the process involved. This is done using a set of scripts that invoke MOSS with all of the current and prior semesters' code as well as any starter code provided to the students. The scripts then collect the results from MOSS, download and "scrape" the HTML pages that MOSS provides, and generate output data files in a format that is amenable to further processing. This step can take up to three hours for a class with approximately 300 student submissions. One interesting complication is that one of the project parts is now so large that we must break up the submission process incrementally and submit the current semester submissions against prior submissions grouped by year - otherwise, MOSS fails to provide results - a frustrating type of error.

Once a case is identified, we review each of the suspect cases to eliminate those that do not appear to be genuine acts of plagiarism. This involves a review of the code and the student's README file. If these indicate a likely case of plagiarism, either a meeting is scheduled with the student - if this is the first time we have observed an issue for this student - or we refer the student to the university. Since the entire program is online, student meetings in this context are via an online video conferencing service. At least two members of the instructional team are present and, with the student's permission, the meeting is recorded. The information from MOSS is presented to them, and we discuss the similarity. If they admit to plagiarism, we give them a grade of zero points on the suspected sections of the project. The outcome is reported to the university, which tracks the issue so that repeated offenses can be handled appropriately while safeguarding the students' privacy rights.

In cases where students ignore our request for a meeting (which frequently happens), tell us they have withdrawn from the course, or do not admit to the plagiarism, we process a formal referral to the university. The referral includes a copy of the plagiarism quiz, the syllabus explaining the course policy, and the code identified by 
Table 4: "Collaboration versus Cheating” Assessment T-Test Results

See §2.3. One-tailed two-sample t-test, assuming unequal variances: demonstrates statistical significance of intervention with respect to pre-intervention classes.

\begin{tabular}{cccc}
\hline Project & T-crit & T-stat & $\mathrm{P}(\leq \mathbf{0 . 0 5})$ \\
\hline PR1-A & 2.23 & 0.03 & $\mathbf{0 . 0 3 2}$ \\
PR1-B & 2.90 & 1.89 & $\mathbf{0 . 0 1 1}$ \\
PR1-C & 2.83 & 1.94 & $\mathbf{0 . 0 1 5}$ \\
PR1-D & 3.93 & 1.94 & $\mathbf{0 . 0 0 4}$ \\
PR2-A & 4.70 & 2.13 & $\mathbf{0 . 0 0 2}$ \\
PR2-B & 4.09 & 1.89 & $\mathbf{0 . 0 0 4}$ \\
\hline
\end{tabular}

MOSS. Once a decision is made, the instructional team is notified. If a grade change is required, a paper form must be completed and signed by various members of the department. Overall, we estimate that prosecuting a case requires more than five hours of instructional team time.

Even the most superficial analysis indicates that any approach that minimizes plagiarism is an improvement over the simple enforcement model. Thus, we defer an actual cost calculation for this improvement.

\section{ANALYSIS}

We are aware of several open questions that relate directly to the veracity of our results.

\subsection{Hidden Plagiarism}

One possible explanation for the observed decrease in the plagiarism rate is that students became better at hiding their plagiarism. In an attempt to look for more subtle forms of plagiarism, we have supplemented our work with MOSS by adding a number of other mechanisms, including code watermarks and SHA-2 checksums, which detect tampering with the code testing environment. We have investigated several suspicious cases, but we found no new cases of plagiarism using these mechanisms. A student brilliant enough to spoof SHA-2 checksums is unlikely to need to plagiarize the code for an introductory graduate operating systems course [45].

In the unlikely case that students develop the level of insight necessary to circumvent the various plagiarism checks in the system, they must do so independently of other students, since MOSS detects collaborative plagiarism. Thus, to circumvent MOSS, a student must find a candidate code base and then substantially alter its structure to avoid detection. Even if a student were to actively use MOSS to confirm that their code no longer appeared structurally similar to the original code they plagiarized, they would then need to also make sure that their modified code worked, which in turn means fixing it and debugging problems that arose due to the substantive changes. Our pedagogical goal is to ensure that students understand the underlying mechanisms; this goal is achieved if the student must understand their now modified code base sufficiently well to pass the tests of their code. This effect has been separately noted, particularly with respect to MOSS and programming classes [20].
Table 5: Unique number of students detected per semester

\begin{tabular}{lrr}
\hline Summer 2015 & 21 & 13.8 \\
Fall 2015 & 27 & 16.8 \\
Spring 2016 & 21 & 14.2 \\
Summer 2016 & 20 & 14.1 \\
Fall 2016 & 23 & 13.1 \\
Spring 2017 & 20 & 14.6 \\
Summer 2017 & 4 & 5.6 \\
Fall 2017 & 11 & 5.1 \\
Spring 2018 & 15 & 5.9 \\
Summer 2018 & 12 & 7.0 \\
\hline
\end{tabular}

One intriguing approach to detect such cases is to perform a more substantive analysis of the student submissions. Our testing mechanism saves all student submissions, which would permit us to evaluate the history of student code submissions more thoroughly using existing techniques [55].

\subsection{Demographic Shifts}

One insightful reviewer of our research suggested that our results might be due to shifting demographics. We do not have demographics for the class, but we do have them for the program (see $\S 1$ ). While there has been a small change in demographics, it seems unlikely that the effectiveness of our approach is due to a modest $(10 \%)$ change in program demographics.

\subsection{Work for Hire}

A reviewer of our research indicated we did not address work-forhire-style cheating solutions. We agree that this is a weakness of our work. We are encouraged by work being conducted by our colleagues within the program to build tools for identifying such code. It also occurs to us that their work might be combined with the analysis-over-time approach suggested in $\S 4.1$ on the theory that a student who contracts out that work would likely only submit it a few times on their own. We can also track the IP address used to submit student work to detect cases where the work-for-hire is given access to the student's account for the purposes of submitting their work to our automatic grading system.

However, we argue that our own work is a valuable tool for decreasing the rate of plagiarism, even if it does not completely eliminate plagiarism.

\subsection{Strict Enforcement}

One possible alternative explanation for the efficacy of our approach is the more rigorous enforcement model. While we have not formally proven this is not a factor, from Summer 2017 through Summer 2018 our enforcement was not as rigorous as it was in Spring 2017. Since Summer 2018 we have made significant strides in automating the process of generating detailed descriptions of our findings, which has made contacting students and preparing referrals to the university less time-consuming. Our scripts anonymize the MOSS output by replacing student names with unique identifiers, convert the HTML pages to PDF documents, and construct one directory per current-semester student with all the relevant 
information. Our hope is this will further reduce our plagiarism rate.

\section{PRIOR WORK}

The basic concepts around plagiarism are hardly new and have been extensively reviewed [27, 28, 37]. Indeed, the literature contains clear discussions on this problem with respect to programming courses decades ago [24]. While we still do not fully understand online-instruction dynamics, the available evidence suggests that plagiarism occurs in both online and in-person programs $[4,15,25,34,41,46,54,57]$. While much of the prior work focused on undergraduate academic dishonesty, we did not find any prior work suggesting that these findings do not apply to coursebased graduate programs as well.

Similarly, the idea of clarifying the expectations for students in programming classes is not new [21, 49]. Our research augments this prior work by evaluating these techniques in an actual classroom setting over a period of 10 semesters.

Motivating students to adhere to academic honesty policies and the instructional strategies that effectively achieve this goal, particularly with respect to plagiarism in computer science classes, remains an open area of research and practice [14]. Although some previous studies have explored the root-cause analysis for plagiarism, our goal is not to understand why students plagiarize, but rather to understand how to discourage it through a combination of detection and education mechanisms [18]. Our approach is consistent with prior studies suggesting a move away from a moralistic view of plagiarism, instead reframing discussion on plagiarism as an integral part of the learning process. In other words, even if students do not understand what plagiarism is when starting the program, educating them about it is important and beneficial, albeit a secondary goal to the instructors' [1]. That said, our research also supports the prior observation that simply informing students about an honor code does not lead to a decrease in plagiarism in online programs [36]. Active education on academic honesty through formal training, however, may be effective [13].

We admit that we do not know the optimal mechanism for motivating students to adhere to academic honesty policies. We have not explored the specific issue of motivations behind plagiarism (which the literature suggests can be cultural, economic, or perceptionbased), but we do note that our successful interventions are consistent with educating students, regardless of their cultural background $[3,7,11]$.

There is a strong body of research describing techniques for reducing plagiarism, but there is a much smaller pool of evaluations regarding the application of these techniques [19, 35, 51]. One side-effect of this paucity of research is that the most common strategy for combating plagiarism is to shift responsibility to individual instructors, which results in haphazard enforcement and poor knowledge-sharing. By providing a rigorous review of techniques applied over time to the same class, with similar student populations, we contribute solid evidence of techniques that work. We hope that universities can incorporate this evidence into a broader initiative toward improving all courses, rather than relying on anecdotal knowledge shared haphazardly between individual instructors $[40,50]$.

\section{FUTURE WORK}

Besides the obvious possibilities raised during our analysis (see §4), we can see several areas of future work:

- We can augment the mechanisms available for detecting plagiarism. While MOSS is an excellent tool, it is not necessarily applicable to all programming classes. Evaluating new mechanisms for detecting code plagiarism will be useful and is an active area of research [5, 6, 16, 17, 26, 30, 33, 38, 39, 53, 56].

- We can work on identifying new ways to modify the behavior of students to achieve our goals of further improving academic honesty.

- We can improve our own analysis of the output using our existing tools. Our work on this project has already encouraged us to develop several tools we can use to ease the evaluation of student code in the future, and we suspect there is further work to be done in this area, particularly with an eye toward simplifying the process for others. Similar augmentations over MOSS have been implemented by others as well $[47,55]$

- We could explore why this approach works. We speculate that it is because of the context in which students are presented with the information - it is presented as a formal quiz, which underscores its importance to students, whereas posts in Piazza are more likely to be viewed as background noise. Studying different ways of presenting this information to the class and measuring the outcome might be possible, but it may also raise ethical research considerations.

We also realize that future classes may learn of the techniques that the course instructors use to detect plagiarism. As a result, course instructors will need to find ways to further improve their detection abilities. At some point, the work required to circumvent these checks becomes greater than the effort of simply doing the assignment - and hopefully achieves the instructors' pedagogical goal [20].

\section{CONCLUSION}

Prior work demonstrates that honor codes alone are not an effective mechanism for reducing code plagiarism; this research demonstrates that explaining and reinforcing lessons in academic honesty results in statistically significant $(p<0.05)$ decreases in plagiarism rates across all of the distinctive programming submissions in a large online graduate computer science course. In addition, this technique requires minimal effort from the instructors - in contrast to a strict enforcement policy, which substantially increases the burden on the instructional staff.

\section{REFERENCES}

[1] Lee Adam, Vivienne Anderson, and Rachel Spronken-Smith. 2017. "It's not fair": policy discourses and students' understandings of plagiarism in a New Zealand university. Higher Education 74, 1 (2017), 17-32.

[2] Alex Aiken. 1994. MOSS: A System for Detecting Software Similarity. http: //theory.stanford.edu/ aiken/moss/ (Accessed January 6, 2018).

[3] Ruth Baker-Gardner and Cherry-Ann Smart. 2017. Ignorance or Intent?: A Case Study of Plagiarism in Higher Education among LIS Students in the Caribbean. In Handbook of Research on Academic Misconduct in Higher Education. IGI Global, 182-205.

[4] Russell Boyatt, Mike Joy, Claire Rocks, and Jane Sinclair. 2014. What (Use) is a MOOC?. In The 2nd international workshop on learning technology for education in cloud. Springer, 133-145. 
[5] Aylin Caliskan-Islam, Richard Harang, Andrew Liu, Arvind Narayanan, Clare Voss, Fabian Yamaguchi, and Rachel Greenstadt. 2015. De-anonymizing programmers via code stylometry. In 24th USENIX Security Symposium (USENIX Security), Washington, $D C$.

[6] Aylin Caliskan-Islam, Fabian Yamaguchi, Edwin Dauber, Richard Harang, Konrad Rieck, Rachel Greenstadt, and Arvind Narayanan. 2015. When coding style survives compilation: De-anonymizing programmers from executable binaries. arXiv preprint arXiv:1512.08546 (2015)

[7] Shih-Chieh Chien. 2017. Taiwanese College Students? Perceptions of Plagiarism Cultural and Educational Considerations. Ethics \& Behavior 27, 2 (2017), 118-139.

[8] Beth Cook, Judy Sheard, Angela Carbone, Chris Johnson, et al. 2014. Student perceptions of the acceptability of various code-writing practices. In Proceedings of the 2014 conference on Innovation \& technology in computer science education ACM, 105-110.

[9] Henry Corrigan-Gibbs, Nakull Gupta, Curtis Northcutt, Edward Cutrell, and William Thies. 2015. Deterring cheating in online environments. ACM Transactions on Computer-Human Interaction (TOCHI) 22, 6 (2015), 28.

[10] Henry Corrigan-Gibbs, Nakull Gupta, Curtis Northcutt, Edward Cutrell, and William Thies. 2015. Measuring and maximizing the effectiveness of honor codes in online courses. In Proceedings of the Second (2015) ACM Conference on Learning@Scale. ACM, 223-228.

[11] Georgina Cosma, Mike Joy, Jane Sinclair, Margarita Andreou, Dongyong Zhang Beverley Cook, and Russell Boyatt. 2017. Perceptual comparison of source-code plagiarism within students from UK, China, and South Cyprus higher education institutions. ACM Transactions on Computing Education (TOCE) 17, 2 (2017), 8.

[12] Victoria L Crittenden, Richard C Hanna, and Robert A Peterson. 2009. The cheating culture: A global societal phenomenon. Business Horizons 52, 4 (2009), 337-346.

[13] Guy J Curtis, Bethanie Gouldthorp, Emma F Thomas, Geraldine M O’Brien, and Helen M Correia. 2013. Online academic-integrity mastery training may improve students' awareness of, and attitudes toward, plagiarism. Psychology Learning \& Teaching 12, 3 (2013), 282-289.

[14] Cesur Dagli. 2017. Relationships of first principles of instruction and student mastery: A MOOC on how to recognize plagiarism. Ph.D. Dissertation. Indiana University.

[15] Charlie Daly and Jane Horgan. 2005. Patterns of plagiarism. In ACM SIGCSE Bulletin, Vol. 37. ACM, 383-387.

[16] Tomche Delev and Dejan Gjorgjevikj. 2017. Comparison of string matching based algorithms for plagiarism detection of source code. (2017).

[17] Xuliang Duan, Mantao Wang, and Jiong Mu. 2017. A Plagiarism Detection Algorithm based on Extended Winnowing. In MATEC Web of Conferences, Vol. 128. EDP Sciences, 02019.

[18] Sarah E Eaton, Melanie Guglielmin, and Benedict Otoo. 2017. Plagiarism: Moving from punitive to pro-active approaches. (2017).

[19] Helen Ewing, Ade Anast, and Tamara Roehling. 2016. Addressing plagiarism in online programmes at a health sciences university: a case study. Assessment \& Evaluation in Higher Education 41, 4 (2016), 575-585.

[20] genchang1234. 2015. genchang1234/How-to-cheat-in-computer-science-101. https://github.com/genchang1234/How-to-cheat-in-computer-science-101 (accessed January 6, 2018)

[21] J Paul Gibson. 2009. Software reuse and plagiarism: A code of practice. In In 14th ACM SIGCSE Annual Conference on Innovation and Technology in Computer Science Education(ITiCSE 2009. ACM, 55-59.

[22] Inc. GitHub. 2018. Guide to Submitting a DMCA Takedown Notice. https://help. github.com/articles/guide-to-submitting-a-dmca-takedown-notice/ (Accessed January 20, 2018).

[23] Joshua Goodman, Julia Melkers, and Amanda Pallais. 2017. Can Online Delivery Increase Access to Education? (2017).

[24] Sam Grier. 1981. A Tool That Detects Plagiarism in Pascal Programs. In Proceedings of the Twelfth SIGCSE Technical Symposium on Computer Science Education (SIGCSE '81). ACM, New York, NY, USA, 15-20. https://doi.org/10.1145/800037. 800954

[25] Therese C Grijalva, Clifford Nowell, and Joe Kerkvliet. 2006. Academic honesty and online courses. College Student fournal 40, 1 (2006).

[26] Daniël Heres. 2017. Source Code Plagiarism Detection using Machine Learning. Master's thesis.

[27] Rebecca Moore Howard. 1995. Plagiarisms, authorships, and the academic death penalty. College English 57, 7 (1995), 788-806.

[28] Rebecca Moore Howard. 1999. Standing in the shadow of giants: Plagiarists, authors, collaborators. Number 2. Greenwood Publishing Group.

[29] Stack Exchange Inc. 2018. Stack Overflow - Where Developers Learn, Share, \& Build Careers. stackoverflow.com (Accessed January 21, 2018).

[30] Hasan M Jamil. 2017. Automated personalized assessment of computational thinking MOOC assignments. In Advanced Learning Technologies (ICALT), 2017 IEEE 17th International Conference on. IEEE, 261-263.

[31] David A Joyner, Ashok K Goel, and Charles Isbell. 2016. The Unexpected Pedagogical Benefits of Making Higher Education Accessible. In Proceedings of the Third (2016) ACM Conference on Learning@ Scale. ACM, 117-120.
[32] Brian W Kernighan and Dennis M Ritchie. 2006. The C programming language. [33] Hiroshi Kikuchi, Takaaki Goto, Mitsuo Wakatsuki, and Tetsuro Nishino. 2015. A source code plagiarism detecting method using sequence alignment with abstract syntax tree elements. International fournal of Software Innovation (IFSI) 3, 3 (2015), 41-56.

[34] Lisa M. Krieger. 2016. Stanford finds cheating âĂŤ especially among computer science students âĂŤ on the rise. https://tinyurl.com/yan2sull (accessed January 6, 2017) originally published February 6, 2010

[35] Bruce Macfarlane, Jingjing Zhang, and Annie Pun. 2014. Academic integrity: a review of the literature. Studies in Higher Education 39, 2 (2014), 339-358.

[36] David F Mastin, Jennifer Peszka, and Deborah R Lilly. 2009. Online academic integrity. Teaching of Psychology 36, 3 (2009), 174-178.

[37] Hermann A Maurer, Frank Kappe, and Bilal Zaka. 2006. Plagiarism-a survey. (2006).

[38] Xiaozhu Meng. 2016. Fine-grained binary code authorship identification. In Proceedings of the 2016 24th ACM SIGSOFT International Symposium on Foundations of Software Engineering. ACM, 1097-1099.

[39] Xiaozhu Meng, Barton P Miller, and Kwang-Sung Jun. 2017. Identifying multiple authors in a binary program. In European Symposium on Research in Computer Security. Springer, 286-304.

[40] Holmes Miss and J Evelyn. 2017. Development and Leadership of a Faculty-led Academic Integrity Education Program at an Ontario College. (2017)

[41] Hannah Natanson. 2017. More than 60 Fall CS50 Enrollees Faced Academic Dishonesty Charges. http://www.thecrimson.com/article/2017/5/3/ cs50-cheating-cases-2017/ (Accessed January 6, 2018)

[42] University of Ontario. 2016. Why is Academic Integrity and Honesty Important? https://secure.apa.uoit.ca/academic_integrity/module1/Module13.html

[43] Inc. Piazza. 2017. Why Piazza Works. https://piazza.com/product/overview Accessed December 31, 2017.

[44] Jonathan Pierce and Craig Zilles. 2017. Investigating Student Plagiarism Patterns and Correlations to Grades. In Proceedings of the 2017 ACM SIGCSE Technical Symposium on Computer Science Education (SIGCSE '17). ACM, New York, NY, USA, 471-476. https://doi.org/10.1145/3017680.3017797

[45] Zulfany Erlisa Rasjid, Benfano Soewito, Gunawan Witjaksono, and Edi Abdurachman. 2017. A review of collisions in cryptographic hash function used in digital forensic tools. Procedia Computer Science 116 (2017), 381-392.

[46] Heather B Shapiro, Clara H Lee, Noelle E Wyman Roth, Kun Li, Mine ÇetinkayaRundel, and Dorian A Canelas. 2017. Understanding the massive open online course (MOOC) student experience: An examination of attitudes, motivations, and barriers. Computers \& Education 110 (2017), 35-50.

[47] Dana Sheahen and David Joyner. 2016. TAPS: A MOSS Extension for Detecting Software Plagiarism at Scale. In Proceedings of the Third (2016) ACM Conference on Learning@ Scale. ACM, 285-288.

[48] Judy Sheard, Michael Morgan, Andrew Petersen, Amber Settle, Jane Sinclair, Gerry Cross, Charles Riedesel, et al. 2016. Negotiating the Maze of Academic Integrity in Computing Education. In Proceedings of the 2016 ITiCSE Working Group Reports. ACM, 57-80.

[49] Simon, Judy Sheard, Michael Morgan, Andrew Petersen, Amber Settle, and Jane Sinclair. 2018. Informing Students About Academic Integrity in Programming. In Proceedings of the 20th Australasian Computing Education Conference (ACE '18). ACM, New York, NY, USA, 113-122. https://doi.org/10.1145/3160489.3160502

[50] Raghu Naath Singh and David Hurley. 2017. The Effectiveness of Teaching and Learning Process in Online Education as Perceived by University Faculty and Instructional Technology Professionals. Journal of Teaching and Learning with Technology 6, 1 (2017), 65-75.

[51] Alison Smedley, Tonia Crawford, and Linda Cloete. 2015. An intervention aimed at reducing plagiarism in undergraduate nursing students. Nurse education in practice 15, 3 (2015), 168-173.

[52] Sami W Tabsh, Hany A El-Kadi, and A Abdelfatah. 2015. Past and present engineering studentsâĂŹ views on academic dishonesty at a middle-eastern university. International fournal of Engineering Education 31, 5 (2015), 13341342.

[53] RL Victor and Farica P PUTRI. 2016. A Code Plagiarism Detection System Based on Abstract Syntax Tree and a High Level Fuzzy Petri Net. DEStech Transactions on Materials Science and Engineering mmme (2016).

[54] Yoav Yair. 2014. I saw you cheating. ACM Inroads 5, 3 (2014), 36-37.

[55] Lisa Yan, Nick McKeown, Mehran Sahami, and Chris Piech. 2018. TMOSS: Using Intermediate Assignment Work to Understand Excessive Collaboration in Large Classes. In Proceedings of the 49th ACM Technical Symposium on Computer Science Education (SIGCSE '18). ACM, New York, NY, USA, 110-115. https: //doi.org/10.1145/3159450.3159490

[56] Xinyu Yang, Guoai Xu, Qi Li, Yanhui Guo, and Miao Zhang. 2017. Authorship attribution of source code by using back propagation neural network based on particle swarm optimization. PloS one 12, 11 (2017), e0187204.

[57] Youdan Zhang. 2013. Benefiting from MOOC. In EdMedia: World Conference on Educational Media and Technology. Association for the Advancement of Computing in Education (AACE), 1372-1377. 